\title{
AIR COMPRESSION SYSTEMS WITH HYDRAULIC DYNAMIC ENERGY
}

\author{
Vallabhaneni Yaswanth ${ }^{1}$, Karrothu V R Manikanta ${ }^{2}$, V. Vinod ${ }^{3}$, D. Prasad $^{4}$ \\ ${ }^{\text {I} B . T e c h, ~ M e c h a n i c a l ~ d e p t ., ~ G u r u ~ N a n a k ~ I n s t i t u t e ~ o f ~ T e c h n o l o g y, ~ H y d e r a b a d, ~ I n d i a ~}$ \\ ${ }^{2}$ B.Tech, Aeronautical dept., Guru Nanak Institutions Technical Campus, Hyderabad, India \\ ${ }^{3}$ B.Tech, Mechanical dept., Guru Nanak Institute of Technology, Hyderabad, India \\ ${ }^{4}$ B.Tech, Mechanical dept., Guru Nanak Institute of Technology, Hyderabad, India
}

\begin{abstract}
A compressor is a machine that capable of compressing and delivering the air at a desired pressure value. It is driven with a prime mover. Air compressor takes in atmospheric air, compresses and delivers to a storage tank. There are many principles of compressors up to now, here we are introduced another principle of air compression system with hydraulic dynamic energy which may be used alternative to all compressors because of its advantages. In order to satisfy these new responsibilities, maintenance, operations, and engineering personnel need continuous review of compressor types, classifications, and applications. HACS overcomes disadvantages of reciprocating compressor for the same pressure produced, like vibrations, discharge air temperature, lubrication and noise. In HACS crank mechanism is eliminated and diving force for the motion of piston is done by pumping the incompressible liquid into the cylinder.
\end{abstract}

Keywords: Compressor, Dynamic energy, HACS, Machine and Piston.

\section{INTRODUCTION}

A hydraulic air compressor (HAC), is a simple, efficient device for compressi.ng air, by means of the energy in a head of water. It is an ancient device, not much used today because motor driven compressors are generally cheaper. Its isothermal compression cycle is ideally suited for combination with a gas turbine. The net saving by combining a hydro site with a peaking gas turbine can be as high as $50 \%$ of the gas turbine capacity. Our proposed principle is differ with the ancient device as our system deals with the utilization of dynamic hydraulic energy to compress air.

\subsection{Types of Air Compressor}

According to the design and principle of operation compressors are as follows

1. Reciprocating compressor

2. Rotary compressor

Among the above compressors reciprocating compressor is most commonly used, for low discharge and high pressures In reciprocating compressor, the compression carried out by containing air between the cylinder walls and the piston and reducing the volume by the moment of the piston.

The main disadvantages of reciprocating compressor are

1. Vibrations due to crank mechanism

2. High temperature of delivery air, to overcome it external cooling is required

3. Lubrication is required for piston moment.

4. Noise produced due to reciprocating and rotary parts
Above disadvantages can be overcome with HACS for the same pressure produced by eliminating the crank mechanism, for the moment of piston hydraulic energy is used i.e., by pumping the liquid into the cylinder

\section{PROPOSED PRINCIPLES}

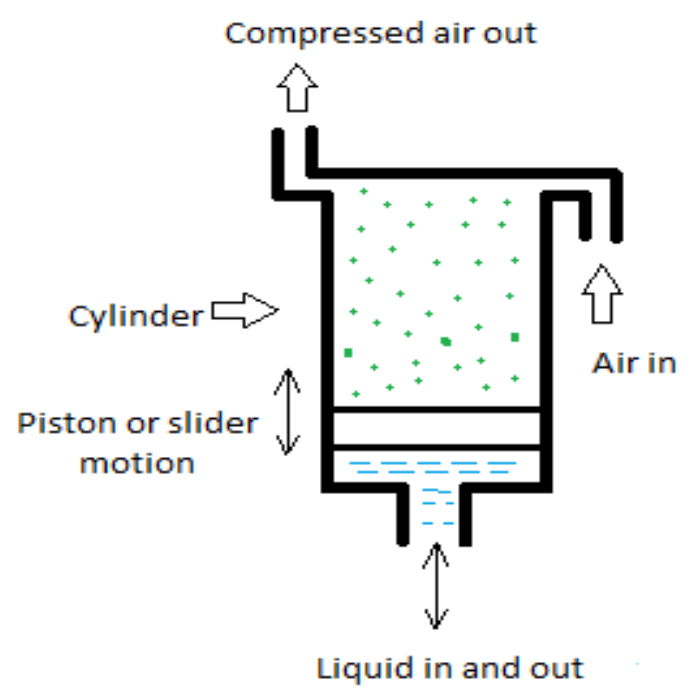

Fig.2.1 HACS principle

In HACS liquid is pumped into the cylinder below the slider that moves the piston upward with pressure equal to liquid pumping in, the compression is carried out in the closed boundary between slider and cylinder walls. When the liquid is draining out slider moves down and air will sucked in. 


\subsection{Principle 1 (Application of HACS where High} Discharge Pump Available)

HACS can be adopted for reciprocating compressors, where the pump available with high discharge and low pressure and the process will be as follows

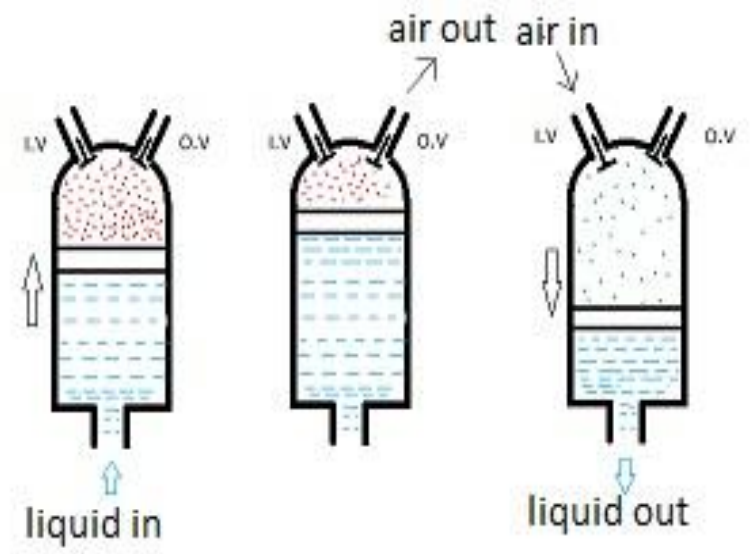

Fig 2.2a Compression Fig 2.2b Delivery Fig 2.2c: Suction

A cylinder is housing the slider that it forms an isolated system and an inlet valve and outlet valve will control the flow of air in and out of cylinder. An incompressible liquid is pumped into the cylinder when slider is at BDC that creates the up word motion of slider as the valves remains closed air will be compressed in the closed boundary as shown in fig 3.1 this is called compression stroke. When piston is at TDC outlet value will be opened and high pressure air is delivered. The liquid will start draining when the discharge completed inlet valve will open and outlet valve will get closed then slider moves downwards because of gravity. Air will be sucked because of the pressure difference between atmosphere and inside of cylinder this stroke is called suction stroke. After suction stroke the process will again repeat.

\subsubsection{Considerations}

1. Discharge and pressure of delivery air is dependent on the dynamic properties of driving liquid

2. Appropriate type of pump should be used on the basis of liquid discharge and pressure

3. By using the same motor for liquid pump and reciprocating compressor at same pressure, discharge will be very low for any type of pump than reciprocating compressor

4. Discharge of liquid pump will makes decide of dimensions of cylinder and it affects the discharge of air

\subsubsection{Theory and Calculations}

As the discharge and pressure of delivery air depends on the dynamic properties of circulating liquid, pump specifications plays a major role in HACS so the comparison of various pumps is given below
Table 1

\begin{tabular}{|l|l|l|}
\hline Type of Pump & $\begin{array}{l}\text { Maximum Pressure } \\
\text { (bar) }\end{array}$ & $\begin{array}{l}\text { Maximum } \\
\text { Flow } \\
(1 / \mathrm{min})\end{array}$ \\
\hline \hline Centrifugal & 20 & 3000 \\
\hline \hline Gear & 175 & 300 \\
\hline Vane & 175 & 500 \\
\hline \hline $\begin{array}{l}\text { Axial Piston - port- } \\
\text { plate }\end{array}$ & 300 & 500 \\
\hline \hline Axial Piston -valved & 700 & 650 \\
\hline \hline In-line Piston & 1000 & 100 \\
\hline
\end{tabular}

Power required to drive the pump can be obtain from the following formula:

$$
P_{H P}=q p / 1714
$$

Where

$P_{H P}=$ horsepower $(H P)$

$q=$ required pump capacity $(\mathrm{gpm})$

$p=$ required pressure $(p s i)$

For example we need to compress the air using HACS to $10 \mathrm{bar}$ then the required power of motor to drive the pump can be calculated as

Required pressure $\mathrm{p}=10 \mathrm{bar}=145 \mathrm{psi}$

Required discharge $\mathrm{q}=60 \mathrm{l} / \mathrm{min}=15.9 \mathrm{gpm}$

Power required $\mathrm{P}=\mathrm{p}^{*} \mathrm{q} / 1714$

$=145^{*} 15.9 / 1714$

$=1.13 \mathrm{hp}$

\section{Force on the air}

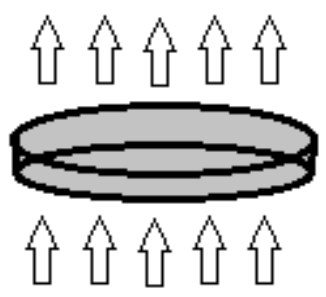

\section{Force by liquid}

Fig 2.3 force on the slider

The area of slider on which force acting by liquid is equal to the area of slider that compresses the air, so the pressure of air delivered is equal to the pressure of liquid pumped into the cylinder. 
The above statement can be proved by following formula

$\mathrm{F}_{1}=$ force on slider by liquid

$\mathrm{F}_{2}=$ force by slider on air

$\mathrm{P}_{1}=$ pressure of liquid

$\mathrm{P}_{2}=$ pressure of air

$\mathrm{D}=$ diameter of slider

$\mathrm{F}_{1}=\left(\pi \mathrm{d}^{2} / 4\right) \times \mathrm{p}_{1}$

$\mathrm{F}_{2}=\left(\pi \mathrm{d}^{2} / 4\right) \times \mathrm{p}_{2}$

Since $F_{1}=F_{2}$

$\left(\pi \mathrm{d}^{2} / 4\right) \times \mathrm{p}_{1}=\left(\pi \mathrm{d}^{2} / 4\right) \times \mathrm{p}_{2}$

$\mathrm{p}_{1}=\mathrm{p}_{2}$

Since pressure by the liquid = pressure in the air (neglecting the weight and pressure losses) (1)

\subsection{Principle 2 (Application of HACS where Pump}

with High Pressure and Low Discharge is

\section{Available)}

HACS can be applied for reciprocating compressor for high pressure and high discharges using same power as reciprocating compressor where the pump is available with high pressure and low discharge.

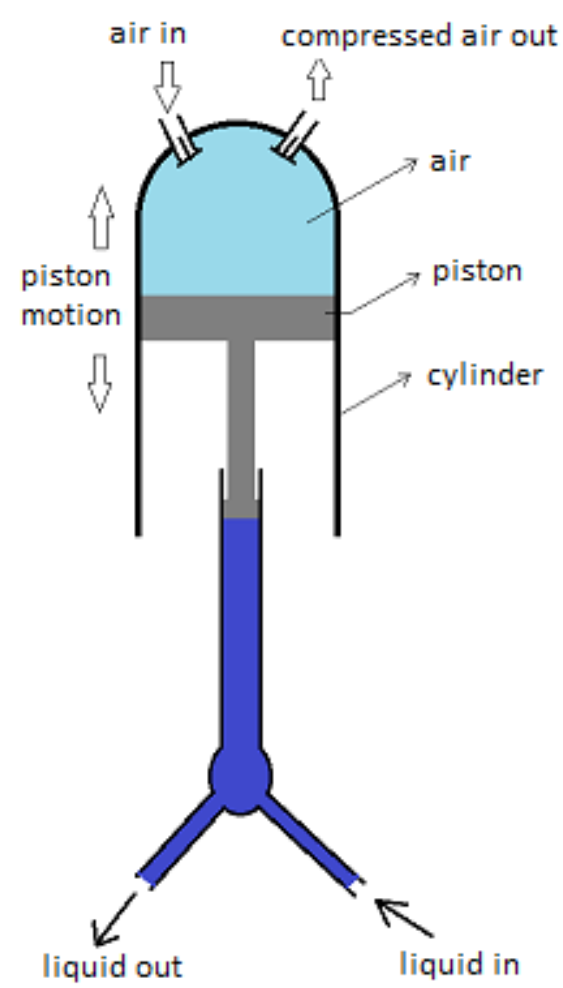

Fig 2.4 HACS for high pressure and low discharge liquid

This is the most important application of HACS for obtaining higher pressure and discharge using HACS the method of applying pressure should be changed. The piston is made of different diameters, the larger diameter is housed in compressing area and smaller diameter is housed in the tube where pressure is applied on the piston as shown in fig4.1
When the high pressure liquid is pumped into the pressure tube it creates high pressure inside the tube that pushes the piston upwards that compresses the air in the cylinder within closed limits as the two valves are closed at that time this is called compression stroke. At the end of compression high pressure air is delivered to receiver through O.V after completion of compression $\mathbf{0 . v}$ will be closed and i.v will open then liquid in the pressure tube will starts draining out that pulls the piston inside the cylinder then air starts sucking into the cylinder due to pressure difference, this is called suction stroke. After suction stroke i.v will be closed and compression stroke will start thus continuing the process

\subsubsection{Piston Nomenclature}

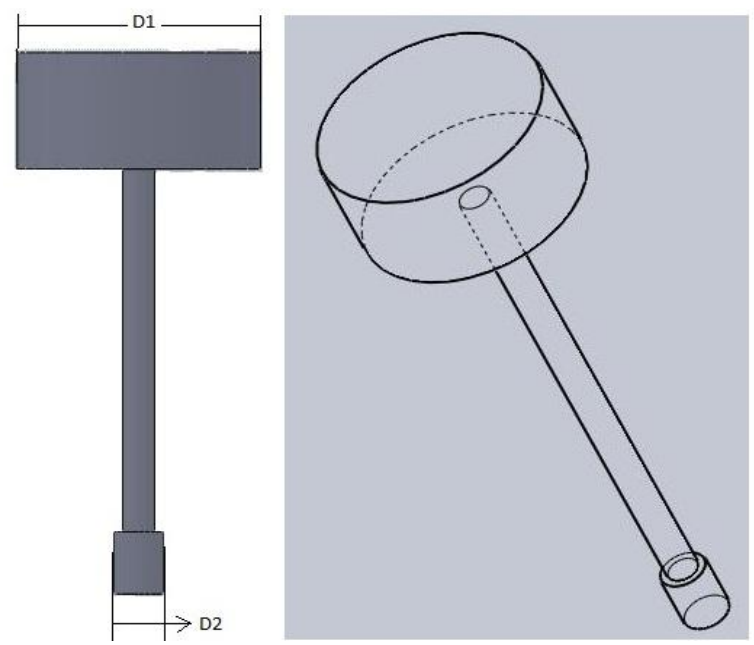

Fig 2.5 Piston

D1 = dia. of piston in the cylinder according to the required air discharge

D2 = dia. of piston in the pressure tube according to the discharge of oil available

Because the discharge of high pressure oil pumps is very low the time taking for filling the liquid in the cylinder will be very high that makes the discharge of compressed air will be very low. So for obtaining high pressures and high discharges the piston should be made of different diameters as shown in fig. Here we apply the pressure on the piston on small diameter as shown in fig 4.2so the low amount oil is enough to compress the air to high pressure

\subsubsection{Calculations}

For testing HACS high pressure discharge take any one of reciprocating compressor available and adopt HACS high pressure system for that compressor for result

Let us take captain brand ME9 model compressor

Motor rating $-3 \mathrm{hp}$

Delivery $-298 \mathrm{l} / \mathrm{min}=0.298 \mathrm{~m}^{3} / \mathrm{min}$

Delivery Pressure - 10bar

Cylinder dimensions - bore X stroke $=98.425$ X 62.25

Speed - $700 \mathrm{rpm}$ 
Adopting HACS for the above compressor using available vane pump

Motor required to drive pump - 3hp

Delivery oil pressure (p1) - 100 bar

Delivery oil discharge $-12.51 / \mathrm{min}=0.0125 \mathrm{~m}^{3} / \mathrm{min}$

In HACS for high pressure and discharge a multi diameter piston as shown in fig 4.2 is used the dimensions of piston are

$\mathrm{D}_{1}=$ same as the bore of cylinder i.e., $98.425 \mathrm{~mm} 100 \mathrm{~mm}$

$\mathrm{D}_{2}$ will be according to the discharge of oil pump

Oil pump discharge $=0.0125 \mathrm{~m}^{3} / \mathrm{min}$

According to oil discharge and cylinder dimensions $\mathrm{D}_{2}=50$ $\mathrm{mm}$

By using the above dimensions the discharge pressure can be calculated as

Force exerted by the oil on the piston

$\mathrm{F}_{1}=\left(\pi \mathrm{d}_{2}^{2} / 4\right) \times \mathrm{p}_{1}=\left(\pi 50^{2} / 4\right) \times 100$

$=196250 \mathrm{~N}$

Since $\mathrm{F}_{1}=\mathrm{F}_{2}$

$196250=\left(\pi \mathrm{d}_{1}^{2} / 4\right) \times \mathrm{p}_{2}$

$196250=\left(\pi 100^{2} / 4\right) \times \mathrm{p}_{2}$

$\mathrm{P}_{2} \quad=25$

$\therefore$ Delivery air pressure will be 25 bar which is more than the pressure of reciprocating pump at same discharge

\section{DISCUSSIONS}

\subsection{For Principle 1}

HACS can be adopted alternatively for reciprocating compressors over same pressure and less discharge for the advantages of

1. Vibrations are minimum

2. No required of cooling system

3. Lubrication not required

4. Low noise levels

By using divergent portion before the cylinder high velocity liquid is converted into high pressure by which we can get more air pressure using centrifugal pump

For compression of air at high pressures and high discharges where high pressure and low discharge pump is available HACS can be adopted by changing the method of apply of pressure on the piston

By adopting HACS where the high discharge pump is available 3 basic models can be made like reciprocating compressors, those are

1. Single cylinder

2. Duel cylinder

3. Single cylinder double acting type

\subsection{For Principle 2}

Advantages of HACS for high pressure discharge are

1. Less vibrations than reciprocating compressor

2. Low noise

3. No separate lubrication system is required, lubrication can be done by a separate loop from the pump

However heat produced during compression is same as reciprocating compressor and can be calculated using adiabatic equation

Note: Calculations are made according to the data available on the internet and assumed that data is genuine. However exact data can be obtained by making experiments.

\section{CONCLUSION}

By observing the structure of proposed principle we can conclude that HACS is practically possible to compress air and theoretical calculations proves that HACS can produce high pressure than any of already existing compressors at the same discharge using same prime mover. Even after leaving the advantage of pressure and discharge which are depends on the liquid pump, advantages of HACS by the design which are indicated in discussions above keeps proposed principle ahead from the existing principles of air compression.

\section{REFRENCES}

[1]. R K Rajput, Thermal engineering, New Delhi: Laxmi Publications, ${ }^{\text {th }}$ Edition (2010).

[2]. Pakirappa, Thermal engineering, New Delhi: Radiant Publishing House. (2002).

[3]. Balje, 0. E., Turbo machines, New York: John Wiley \& Sons, Inc. (1981).

[4]. www.Engineeringtoolbox.com

[5]. Onkar singh, Applied thermodynamics, New age international publishers, third edition (2009). 